\title{
Research on Stroke Rehabilitation Nursing Mode with Senior Nurses as Bond
}

\section{He Xiao-hong ${ }^{a}$ Wang Ping $^{b}$ Rui Zu-qin ${ }^{c}$ Zhang Xiang-lid ${ }^{d}$ Ma Wen-yun ${ }^{e}$ Lu Min $^{f}$}

\author{
Anhui Southern Rehabilitation Hospital No.5 People's Hospital of Wuhu City \\ ${ }^{a}$ https://orcid.org/0000-0001-5175-699X ${ }^{b}$ https://orcid.org/0000-0003-1330-441X \\ c https://orcid.org/0000-0003-3198-9475 d https://orcid.org/0000-0002-0799-4496 \\ e https://orcid.org/0000-0001-8173-6738 f https://orcid.org/0000-0003-0573-8189
}

Received: 12 December 2019 Accepted: 25 December 2019 Published: 31 March 2020

Cite this Article He Xiao-hong, Wang Ping, Rui Zu-qin, Zhang Xiang-li, Ma Wen-yun, Lu Min. Research on Stroke Rehabilitation Nursing Mode with Senior Nurses as Bond [J].Medical Research,2020.2(1):1520, http://dx.doi.org/10.6913/MRHK.202003_2(1).0004

Copyright (C) 2020 Creative Publishing Co., Limited. All rights reserved. Email: kycbshk@gmail.com.

\section{ABSTRACT}

Objective To explore the effect of rehabilitation nursing model for stroke patients with senior nurses.

Methods From October 2017 to October 2018, 46 stroke patients with hemiplegia in community hospitals were selected. 23 stroke patients in community who were willing to accept rehabilitation nursing service package were included in the observation group. The elderly nurses in sinking community were the leading nurses in rehabilitation nursing and home rehabilitation nursing guidance. Community stroke patients without rehabilitation nursing package were selected as control group. Community nurses were the leading nurses in rehabilitation nursing and home-based rehabilitation nursing. The incidence of pressure sores, aspiration rate, accuracy rate of anti-spasm posture placement, and mastery rate of intermittent catheterization technique were compared between the two groups.

Results After the intervention, the incidence of pressure ulcer and aspiration rate in the observation group were significantly lower than those in the control group $(P<0.05)$. The accuracy of anti-spasm posture placement and the mastery rate of intermittent catheterization were significantly higher in the observation group than in the control group $(\mathrm{P}<0.05)$. Conclusion Stroke rehabilitation nursing model with senior nurses as a link has certain effect on rehabilitation nursing of stroke patients in community by strengthening the training, learning and guidance of rehabilitation nursing knowledge and skills of community nurses. Nurses in community hospitals have strengthened two-way linkage, which is of research value, but they are now voluntarily selected in the community. The number of patients choosing rehabilitation care packages is still relatively small, the number of senior nurses in sinking communities is relatively small, and there is still a lot of work to be done to achieve real results. This is a long-term project, which requires time and experience precipitation.

Key words Senior nurses; Link; Stroke; Rehabilitation nursing

Fund Project Task Established by Anhui Provincial Innovative Municipal RMU (October, 2017) 
Regional Medical Union, or RMU in short, which integrates the medical resources in a region, consists usually of third-tier, second-tier, and community hospitals in the region. It aims to solve people's difficulties in going to a doctor and realize the expected people's, the governments, and the employees' satisfaction[1]. Anhui Southern Rehabilitation Hospital chose senior nurses to work in RMUs in the community according to Anhui Province's requirements on pilots of innovative RMUs to optimize the distribution and structure of medical resources, implement better classified receive and treatment system, meet the residents' demands on integrated health services, and make full use of some senior nurses. Stroke is a crucial factor ${ }^{[2-4]}$ that affects the living quality of residents in the community as it is a difficulty in community rehabilitation which has great influence on family and imposes many problems in household nursing. There were 23 patients with hemiplegia caused by stroke selected, who were received by the hospital from Oct, 2017 to Oct, 2018 and hoped to receive rehabilitation nursing and instructions on household rehabilitation nursing with senior nurses leading them to get satisfactory effects. Detailed information is as follows.

\section{DOCUMENTS AND METHODS}

\subsection{General Documents}

There were 46 patients with hemiplegia caused by stroke selected as the object of the research, who were received by the community hospital from Oct, 2017 to Oct, 2018. All the patients matched the relevant diagnosis standards of stroke ${ }^{[5]} .23$ of the stroke patients who hoped to receive service package were grouped as the observation group according to whether they or their families wanted the rehabilitation nursing service package, and would receive the rehabilitation nursing and instructions on household rehabilitation nursing led by the senior nurses of sinking community. There were 15 male and 8 female patients; Their ages ranged from 44 to 86 and their average age was $67.65 \pm 4.78$; 16 of them had cerebral infarction and 7 had cerebral hemorrhage. Their courses of the disease ranged from 3 to 8 months and the

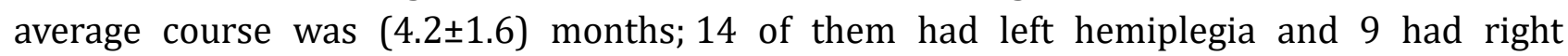
hemiplegia. The other 23 stroke patients in the community who did not choose the service package were grouped in the control group. There were 16 male and 7 female patients; Their ages ranged from 45 to 87 and their average age was $68.26 \pm 5.05 ; 14$ of them had cerebral infarction and 9 had cerebral hemorrhage. Their courses of the disease ranged from 3 to 9 months and their average course was $4.7 \pm 1.8$ months; 16 of them had left hemiplegia and 7 had right hemiplegia. This research observed the ethical requirements and patients or their families signed the informed consent to express their agreement and that they knew the situation. The comparative difference in gender, age, type of stroke, course of the disease, and side of hemiplegia was not statistically significant ( $p>0.05)$ so they were comparable.

\subsection{Methods}

The control group will receive the regular rehabilitation nursing and instructions on household rehabilitation nursing(the rehabilitation service pack) led by the community nurses. The observation group will receive the rehabilitation nursing and instructions on household rehabilitation nursing (the rehabilitation nursing service package) led by the senior nurses. Detailed methods are as follows. (1)The rehabilitation nursing team was established consisting of senior nurses as leading members, senior nurses in community hospitals, 
community nurses, rehabilitation specialist nurses, directors of the task, and staff, with municipal RMUs as supporters, senior nurses as bonds, aiming at groups needing rehabilitation treatment and nursing, and taking continuousness of rehabilitation-treatment and rehabilitation nursing and personalized services as a priority. (2) The role of health-care system in rehabilitation hospitals and community health service centers on management of rehabilitation from diseases must be established by intensifying the leading role of senior nurses and continuous service procedures including initial diagnosis in community-appointed referral-bidirectional referral (community-hospital-community). (3)The items needing training and instructions of implementation during rehabilitation nursing

\subsubsection{Good Limb Posture Placement on Bed}

The nurses must instruct the patients on lying position in the bed, lying position of uninjured side, and limb placement of injured side posture, and explain the functions and necessity of these postures. The injured side posture is very important in good limb posture placement on bed, as it can not only squeeze the trunk on the injured side and promote the recovery of feelings, but also make the patients do some motions on the uninjured side to build their confidence [6]. However, as patients dislike this posture, they must give patients clear explanation. Supination posture tends to cause TLR, but it is indispensable for patients to switch their postures. Therefore, the three postures above-mentioned must be switched according to the patients' conditions. Their postures are suggested to switch every two hours if they need passive placement while the patients switch their postures themselves if they can ${ }^{[7]}$.

\subsubsection{In-bed Movement}

The nurses must instruct the patients to move in the bed when they are in a lying position, with their uninjured feet placed below their injured feet. The uninjured arm fixes the injured arm in front of chest and the uninjured leg lifts the injured leg to move towards the same side. The hip must be supported by both uninjured foot and shoulder and move towards the same side. After the hip has moved to the side, shoulder and head must be moved in the same direction. They must instruct the patients to switch from lying position in the bed to sitting position in the bed according to conditions of the patients' functions.

\subsubsection{Instructions on Choosing and Using Wheelchairs}

The nurses must instruct the patients to choose low-backrest wheelchair as much as they can, do bed-to-wheelchair and wheelchair-to-ed transfers, reduce pressure on the wheelchair, move the wheelchair correctly, and stand under the protection of wheelchair, and etc.

\subsubsection{Training and Instructions of Walking Sticks and Walkers and etc}

When the patient's level of standing balance reaches level 3, they can be instructed to have walking drills. But taking his or her safety into consideration, the nurses must instruct them to use walkers and walking stick correctly.

\subsubsection{Instructions on Household Modification}

For the majority of stroke patients, they will spend large amount of time at home after the occurrence of the disease, but the structure of houses makes the wheelchair fail to pass normally. In addition, patients suffer from much inconvenience in their daily life especially going to restroom and taking a shower without handrail, etc. Therefore, the nurses must instruct them to modify their living circumstance according to their conditions to ensure barrier-free movement[8]. 
If the stroke patient has cardiopulmonary dysfunction, cough, or other diseases involving organs, they must be instructed to conduct respiratory and coughing training. They can also have clean intermittent catheterization if it is necessary for them and they can train part of their muscular strength to prevent muscular atrophy. For example, they can train their musculi quadriceps femoris, ankle pump movement,and etc.

\subsection{Observation Indicators}

The nurses must watch the patients' occurrence of pressure sore and record occurrence rate of pressure sore and aspiration, accuracy rate of anti-spasm posture placement, and patients or their families' mastery rate of intermittent catheterization technique.

\subsection{Statistical Methods}

SPSS 23.0 software was used to perform statistical analysis on the obtained data. Measurement data were expressed as $( \pm s)$, using t test; counting data were expressed as rate (\%), using $\chi 2$ test, $\mathrm{P}<0.05$ was considered statistically significant.

\section{RESULTS}

After the intervention, the occurrence rate of observation group's pressure sore and aspiration is lower than that of control group with statistical significance $(\mathrm{p}<0.05)$. The observation group's accuracy rate of anti-spasm posture placement and the patients or their families' mastery rate of intermittent catheterization technique are both higher than that of control group with statistical significance $(\mathrm{p}<0.05)$.The detailed information can be seen in Table 1 .

Table 1 Comparison of occurrence rate of pressure sore, aspiration rate, accuracy rate of anti-spasm posture placement, and patients or their families' mastery rate of intermittent catheterization technique between the two groups $(\% ; n=23)$

\begin{tabular}{ccccc}
\hline Groups & $\begin{array}{c}\text { Occurrence of } \\
\text { pressure sore }\end{array}$ & $\begin{array}{c}\text { Aspiratio } \\
\text { n rate }\end{array}$ & $\begin{array}{c}\text { Accuracy rate of } \\
\text { anti-spasm posture } \\
\text { placement }\end{array}$ & $\begin{array}{c}\text { Patients or their families' } \\
\text { mastery rate of intermittent } \\
\text { catheterization technique }\end{array}$ \\
\hline $\begin{array}{c}\text { Control } \\
\text { Group }\end{array}$ & $43.48(10 / 23)$ & $\begin{array}{c}47.83(11 / \\
23)\end{array}$ & $52.17(12 / 23)$ & $47.83(11 / 23)$ \\
$\begin{array}{c}\text { Observation } \\
\text { Group }\end{array}$ & $4.35(1 / 23)$ & $8.70(2 / 23)$ & $86.96(20 / 23)$ & $91.30(21 / 23)$ \\
$\chi 2$ & 9.678 & 8.685 & 6.571 & 10.268 \\
P & 0.002 & 0.003 & 0.010 & 0.001 \\
\hline
\end{tabular}

\section{DISCUSSION}

A stroke is an acute medical condition in the brain because blood fails to flow into the brain vessels as a result of sudden crack or vessel block which damage the cerebral tissues[10]. According to relevant investigation[11-12], stroke has been top cause of death and disability among adults in China with its high occurrence rate, mortality rate, and rate of disabilities, leaving severe impact on the patients' living standards. After the stroke patients get out of hospital or rehabilitation center, they receive rehabilitation treatment in the community due to economic reasons or another. However, making and implementing rehabilitation treatment plans relies on three parties, respectively leaders, organizations, people and groups of community, relevant government departments (including health, education, human 

resources, labor force, civil administration, social service departments, and etc), as well as the patients themselves and their families[13]. Only by the union and cooperation of these three parties can the task of rehabilitation in community be completed. At present, effects of community rehabilitation is not very ideal as the professional levels of nurses involving community rehabilitation needs further improvement and leaders lay little stress on it, and etc. This paper conducts the mode through which senior nurses make rehabilitation nursing mode as bonds and enhance the study and instructions on expertise and skills of rehabilitation nursing for the rehabilitation nursing personnel, and effects of rehabilitation nursing on stroke patients in the community have been got. The results show: After the intervention, the occurrence rate of observation group's pressure sore and aspiration is lower than that of control group with statistical significance $(\mathrm{p}<0.05)$. The observation group's accuracy rate of antispasm posture placement and the patients or their families' mastery rate of intermittent catheterization technique are both higher than that of control group with statistical significance $(\mathrm{p}<0.05)$. This reflects that the mode where senior nurses do rehabilitation nursing as bond makes full use of part of senior nurses, and enhances the professional levels of nursing personnel in the community. At the same time, this mode makes the patients easier to master the correct anti-spasm posture placement, increases the patients or their families' mastery rate of intermittent catheterization technique, and avoids the occurrence rate of pressure sore and aspiration. Such mode also enhances the bidirectional interconnectivity of nursing personnel in both community and hospital, satisfying some residents' demands on integrated health services ${ }^{[14]}$.

But there are still disadvantages in such mode: The quantity of patients who hope to receive the rehabilitation nursing service package in the community still remains little; Bidirectional referral and interconnectivity is inadequate; There are inadequate human resources, ages, expertise structures, and updating of expertise among the senior nurses in sinking community; If the nurses have no qualification of professions, they must establish a team with the medical personnel, which will constrain the work. This is a long-term project that needs time and accumulation of experience and there is still a lot to do if we want to get concrete achievements.

\section{REFERENCES}

[1]Huang Pei, Yi Lihua. Practice and Thoughts on Three Different Types of Regional Medical Union [J]. Chinese Hospital Management, 2015,35(2):16-19.

[2]Duan Ruikang, Fu Jianliang. Recognition Disorders After Stroke [J]. Chinese Journal of Gerontology, 2017,37(24):6272-6274.

[3]Gu Hongjun, Su Wei, Ji Yanyu, et al. Clinical Study of Low-frequency Electrical Stimulation Combined with Swallowing Training in the Treatment of Stroke Dysphagia [J]. Rehabilitation in China, 2016,31(4):276-276.

[4]Wang Xiujuan. Effect of Good Limb Placement in Early Rehabilitation Nursing on Rehabilitation of Stroke Patients [J].Modern Journal of Integrated Chinese and Western Medicine,2015,24(15):16881690.

[5]Yang Qinghua, Wu Yuxia, Kong Mezhu, et al. Effect of Community Rehabilitation Combined with Home Environment Transformation on Life Ability of Stroke Patients [J]. Rehabilitation in China, 2017,32(2):168-169.

[6]Li Yaoli. Effects of Systematic Rehabilitation Guidance on Swallowing and Limb Function Recovery in Stroke Patients [J]. Chinese Journal of Gerontology, 
[7]Wang Qiuhua, Wang Li, Zhang Guoli, et al. Study on the Influential Factors of Cognitive Function Recovery in Stroke Patients with Early Community Rehabilitation [J]. Chinese Journal of Cardiovascular and Cerebrovascular Diseases, 2015, 17(3):246-249.

[8]Xie Lijuan, Wang Jianhua, Tian Suzhai, et al. Application of Rehabilitation Guidance Based on Motion Imagination in Stroke Patients with Hemiplegia [J]. Chinese Journal of Modern Nursing, 2016,22(26):3766-3768,3769.

[9]Mao Wei. Preliminary Evaluation on Rehabilitation Effect of Community and Family Rehabilitation Guidance for Stroke Patients [J]. Journal of Cardio-cerebrovascular Diseases Combined with Traditional Chinese and Western Medicine,2017,15(19):2478-2480.

[10]Zhang Ming, Li Shugang, Zhu Qian, et al. Effects of Family Rehabilitation Guidance on Limb Function and Quality of Life of Stroke Patients [J]. Chinese Journal of Clinical Physicians (Electronic Edition),2017,11(19):2533-2258.

[11]Li Xiaoping, Wu Lihong, Ren Tong, et al. Effect of "Hospital-community" Combined Pressure Sore Nursing Group on Continuous Nursing Care After Discharge of Stroke Patients Complicated with Pressure Sore [J]. Chinese General Medicine,2016,19(16):1964-1967,1968.

[12]Wu Shuang, Jing Liwei, Xing Fengmei, et al. Effect of Omaha System's Intervention on Activities of Daily Living and Quality of Life in Elderly Patients with Stroke [J].Chinese Journal of Gerontology, 2017, 37(8):2035-2037.

[13]Lou Lingzhi, Li Jing, Wang Shaowu, et al. Analysis of the Curative Effect of Community Site and Family Integration Rehabilitation for Stroke Patients [J].Shanghai Medicine, 2018, 39(2):57-60.

[14]Tianling, Xie Jiaxing, Zhang Jing, et al. Effect of Community Comprehensive Rehabilitation Management on Quality of Life of Patients with Stroke Sequelae [J]. Theory and Practice of Rehabilitation in China, 2016, 22(12):1467-1470. 